\title{
Comparación del perfil de enoturistas y oleoturistas en España. Un estudio de caso
}

Genoveva Millán Vázquez de la Torre*

Leonor M. Pérez**

Recibido: 2013-09-25 Aprobado: 2014-07-07 Disponible en línea: 2014-07-27

doi:I0.III44/Javeriana.CRDII-74.cpec

Cómo citar este artículo: Millán Vásquez de la Torre, G. y Pérez, L. M. (2014). Comparación del perfil de enoturistas y oleoturistas en España. Un estudio de caso. Cuadernos de Desarrollo Rural, II(74), 165-188. http://dx.doi. org/I0.III44/Javeriana.CRDII-74.cpec

\section{Resumen ${ }^{* * *}$}

Como el vino y el aceite de oliva son los dos sectores agrarios más importantes de España, en muchas regiones se han creado rutas turísticas enfocadas en estos productos para favorecer el desarrollo rural. Este artículo analiza semejanzas entre enoturistas y oleoturistas, a partir de encuestas realizadas en rutas de la provincia de Córdoba (España) con el fin de examinar las posibilidades de comercialización entre ambos grupos. Los resultados muestran semejanzas en características, comportamiento turístico y determinantes del grado de satisfacción. Los resultados sugieren que las distintas industrias (vino, aceite y turismo) podrían trabajar de forma conjunta para promover el desarrollo rural.

\section{Palabras clave:}

desarrollo rural; turismo gastronómico; enoturismo; oleoturismo.

\footnotetext{
* Doctora en Ciencias Económicas, Profesora Titular del Departamento de Economía, Facultad de Ciencias Económicas y Empresariales, Universidad de Córdoba (España). Correo electrónico: gmillan@etea.com ** Profesora Ayudante. Doctora del Departamento de Estadística, Econometría, Investigación Operativa, Organización de Empresas y Economía Aplicada de la Universidad de Córdoba (España).

Correo electrónico: lperez@uco.es

*** Las autoras quieren agradecer el apoyo financiero prestado a esta línea de trabajo por la Consejería de Turismo Comercio y Deporte (Formación, Fomento de la Cultura de la Calidad e Investigación en Materia de Turismo CO-007/08-FFI).
}

\section{c) $\theta \otimes$}




\title{
Comparison of the Profile of Wine and Olive Oil Tourists in Spain. A Case Study
}

\begin{abstract}
As wine and olive oil are the two most important agricultural sectors in Spain, in many regions tourist routes focused on these products have been established to promote rural development. This article analyzes similarities between wine and olive oil tourists from surveys conducted in routes of the province of Córdoba (Spain), in order to examine the commercialization possibilities between the two groups. The results show similarities in characteristics, tourist behavior and determinants of satisfaction degree.The results suggest that the different industries (wine, olive oil and tourism) could work together to promote rural development.
\end{abstract}

Keywords:

rural development; culinary tourism; wine tourism; olive oil tourism

\section{Comparaison du profil d'oenotouristes et de touristes visitant les champs d'oliviers en Espagne. Une étude de cas}

\section{Résumé}

Comme le vin et l'huile d'olive sont les deux secteurs agraires les plus importants d'Espagne, des routes touristiques se sont créées dans beaucoup de regions focalisées sur ces produits pour favoriser le développement rural. Cet article analyse les similitudes entre les oenotouristes et les touristes visitant les champs d`oliviers, à partir des enquêtes faites aux routes de la province de Córdoba (Espagne), afin d'examiner les possibilités de commercialisation entre les deux groupes. Les résultats montrent des similitudes entre les caractéristiques, le comportement touristique et les déterminants du degré de satisfaction. Les résultats suggèrent que les différentes industries (du vin, de l'huile, et du tourisme) pourraient travailler ensemble pour promouvoir le développement rural.

Mots-clés:

développement rural, tourisme gastronomique, oenotourisme, tourisme dans les champs d'oliviers 


\section{Introducción}

En los últimos decenios el turismo se ha convertido en uno de los sectores económicos con mayor crecimiento y diversificación a escala mundial. Algunos países que ofrecen destinos turísticos rurales han invertido cada vez más en el desarrollo de esta actividad, con el fin de enfrentar el estancamiento económico y prevenir la emigración, iniciativa apoyada por políticas comunitarias. Por ejemplo, la Política Agrícola Común de la Unión Europea recomendó la aplicación de alternativas o actividades complementarias en el sector agrario (por su parte, el “Objetivo 5b" de la Unión Europea (UE) y los programas Leader señalaron el papel crucial del turismo en la política agrícola, bien por la creación de productos turísticos con base en recursos rurales o bien por el fomento de producción orientada al ocio (Tomljenovic y Getz, 2009). En ambos contextos, el turismo gastronómico basado en productos agrarios de alta calidad ha emergido en muchas regiones como una de las actividades de turismo rural más importantes.

El desarrollo del turismo gastronómico resulta en una simbiosis entre el sector agrario y el sector turismo. Los productos agrícolas de alta calidad se convierten en recursos atractivos que hacen posible el desarrollo del turismo, al mismo tiempo que el turismo se convierte en una herramienta promocional y de marketing para estos productos (Armesto y Gómez, 2004; Kivela y Crotts, 2006). Por otra parte, como los productos agrarios pueden emplear marcas regionales, existe la oportunidad de crear una asociación positiva entre estos y el destino (Okumus, Okumus y McKercher, 2007). Además, los consumidores cada vez están más preocupados por conocer de dónde proceden los productos y cómo son elaborados, no solo por razones de seguridad y salud, sino también para satisfacer su nostalgia por aquellos tiempos cuando los alimentos se consideraban como sanos y auténticos (Gilg y Battershill, 1998).

La existencia de estas tendencias dentro de las actitudes del consumidor impulsó a la UE a implementar regulaciones (2081/92 y 2082/92) para proteger los productos que tienen un carácter especial (producción con materia prima tradicional o bajo modo tradicional de producción); o un origen geográfico reconocible (por ejemplo, la Denominación de Origen Protegida [DOP], o la Indicación Geográfica Protegida [IGP]) (Ilbery y Kneafsey, 2000). Así, muchos de los productos agrarios que se ofertan como atractivos turísticos suelen tener una calidad certificada mediante una DOP o una IGP. De igual forma, suelen crearse rutas gastronómicas en un espacio limitado que por lo general coincide con una región alimentaria demarcada de manera oficial o con una indicación geográfica (Bruwer, 2003). Un ejemplo es el caso de Champagne, ruta del vino en Francia (Charters y Spielmann, 2014). 
En general, la constitución de una DOP ha sido fundamental en determinadas zonas agrícolas y ha constituido una oportunidad para el desarrollo turístico (Scherrer, Alonso y Sheridan, 2009). No hay duda de que el desarrollo de este tipo de turismo ofrece una serie de beneficios a los destinos rurales a escalas tanto individuales como colectivas, lo que incluye el aumento del número de turistas, la mejora de la imagen del destino y el incremento de las ventas en el propio lugar de producción (Tomljenovic y Getz, 2009).

Asimismo, como afirman Hojman y Hunter-Jones (2012), la visita al lugar de producción (bodegas o almazaras) ofrece una oportunidad única para iniciar una relación con los clientes, pues su experiencia no se limita al momento de la visita, sino que incluye el consumo del producto cuando el turista vuelve a casa. Además, propicia la venta en el lugar de origen del turista, en especial, si es un producto agrario de alta calidad. Hay que agregar que en muchas regiones este turismo gastronómico es una actividad económica desestacionalizada (Miranda y Fernández, 20II), por tanto, se convierte en un complemento interesante del turismo rural tradicional.

Sin embargo, el impulso de este tipo de actividad económica también implica costes significativos para las empresas agrarias que, de forma particular, deciden embarcarse en su desarrollo y en generar infraestructuras de apoyo y acciones de marketing apropiadas (Tomljenovic y Getz, 2009). Emprender este tipo de turismo depende, en general, no solo de acciones independientes por parte de empresarios agrarios, sino de políticas y programas de organismos gubernamentales, así como del marketing de organizaciones de turismo o del destino. De este modo, el establecimiento de una alianza entre los diferentes actores puede desempeñar un papel estratégico en el apoyo a estas iniciativas (Hall, 2005a). Macionis y Cambourne (1998), al estudiar los vínculos estratégicos en el turismo del vino, concluyeron que si se desarrollan de forma activa los vínculos centrados en las necesidades del consumidor, y si se identifican oportunidades de promoción cruzada, el sector del vino y el sector turístico pueden generar una fuerte imagen culinaria en el ámbito regional y aumentar el número de turistas.

Estudios previos analizan el papel de las alianzas que se suelen crear en torno a una ruta alimentaria. Por ejemplo, la iniciativa "Tastes of Niagara” (Telfer, 2000), en la región del vino de Niágara (Canadá), demuestra cómo una alianza estratégica establecida entre distintos entes (productores de alimentos, procesadores, distribuidores, hoteles, bodegas, restaurantes y chefs) puede fomentar el desarrollo de la zona cuando trabajan en conjunto para promover el consumo de productos locales en la industria turística (Telfer, 200I). 
La creación de una ruta gastronómica, como elemento potenciador de desarrollo en zonas agrícolas, engloba un conjunto de actividades y atracciones turísticas que estimula la cooperación y asociación entre empresas de diferentes sectores, así como entre comunidades en regiones locales y vecinas (Briedenhann y Wickens, 2004). Sin embargo, ¿qué ocurre cuando en una región hay más de una ruta alimentaria? ¿Cuál sería el impacto de establecer alianzas entre estas?

En España, por ejemplo, el vino y el aceite de oliva son los dos subsectores agrícolas más importantes, tanto en términos de alcance geográfico y producción como desde un punto de vista histórico y cultural. Ambos se producen en muchas regiones y se han creado rutas turísticas enfocadas tanto en el vino como en el aceite de oliva. No obstante, en ningún caso se han fomentado acciones conjuntas entre ambas rutas, lo que podría significar el desperdicio de potenciales sinergias. La cuestión es: ¿podría establecerse algún tipo de acción conjunta para favorecer el turismo gastronómico de la zona? ¿ Son los turistas del vino (enoturistas) similares a los turistas del aceite de oliva (oleoturistas), o existen diferencias entre ellos? Si hubiera semejanzas se podrían desarrollar de forma activa vínculos dirigidos a las necesidades del consumidor, y realizar acciones comunes de promoción. A partir de esa premisa, esta investigación busca comparar el perfil de enoturistas y oleturistas en rutas ligadas a denominaciones de origen en la provincia de Córdoba, en España, con el objetivo de identificar semejanzas.

Dentro del turismo gastronómico, la mayoría de las investigaciones aborda las actividades derivadas del vino, y varias se enfocan tanto en la oferta como en la demanda. En lo que respecta a la demanda, tales estudios analizan su caracterización (Mitchell y Hall, 2006); segmentación (Charters y Ali-Knight, 2002); motivación y satisfacción (Bruwer y Alant, 2009); estados (previsita, visita y posvisita) (Mitchell y Hall, 2004); factores (Dodd, 1999); y otros temas (Hall, 2005b). Pero no existen estudios que comparen el perfil del enoturista con el perfil del turista atraído por diferentes productos agrarios.

En España, las investigaciones sobre enoturismo han abarcado básicamente lo relativo a la oferta turística (López-Guzmán, Rodríguez y Vieira, 2013). Diferentes autores (López-Guzmán, Rodríguez y Vieira, 2013; Marzo y Pedraja, 2010) ponen de manifiesto la necesidad de reforzar el estudio de la demanda, aunque existen investigaciones que la analizan en determinadas zonas geográficas como Canarias (Díaz, 2008), el Marco de Jerez (López-Guzmán, Rodríguez y Vieira, 2014) o el Condado de Huelva (Vargas, Porras, Plaza y Riquel, 2008). Además, varios trabajos examinan la potencialidad de la demanda enoturística en Valencia (Clemente, Rodríguez y Buitrago, 2010) y Aragón (Marzo y Pedraja, 2009; 2010; 20I2). 
Por otra parte, la literatura sobre turismo gastronómico referente a otros productos agrarios, como por ejemplo el aceite de oliva, es muy escasa. En oleoturismo, las pocas investigaciones realizadas han abordado la oferta en países como Australia (Duarte, 20го) y España (Millán, Agudo, Morales, 20II), y analizado la demanda potencial de este tipo de turismo (Molina, Quesada y Ruiz, 20II; Millán, Arjona y Amador, 20I4). En resumen, la originalidad de este estudio reside en la necesidad de profundizar en lo que respecta a la demanda de enoturismo y oleoturismo en España, así como en la falta de trabajos de investigación que comparen ambos perfiles.

Para llevar a cabo este trabajo se analizaron datos de una encuesta a 500 enoturistas en la ruta del vino de Montilla-Moriles y a 2324 oleoturistas en las rutas del aceite de oliva de Baena, Montoro-Adamuz y Priego de Córdoba. Los resultados pueden contribuir a que los destinos que deseen fomentar de manera simultánea las actividades de enoturismo y oleoturismo, puedan diseñar planes estratégicos eficaces. El artículo comienza con una breve introducción sobre las rutas gastronómicas en España, en particular, en Córdoba, seguida de la metodología, la descripción de los resultados y, por último, la discusión de estos y las conclusiones.

\section{Rutas gastronómicas en España}

España cuenta con una tradición, que se remonta a la época de los romanos, en lo que concierne a la elaboración de vinos. Es el país con mayor extensión de viñedos del mundo, con I.16 millones de hectáreas destinadas al cultivo de la vid, de las que 97.4\% se destina a vinificación (Organización Internacional de la Viña y el Vino, 20I4). La producción en la campaña 20I3-20I4 ascendió a 52.6 millones de hectolitros, la cifra más elevada históricamente y que le convierte en el primer país del mundo en producción vitivinícola (Fondo Español de Garantía Agraria, 20I4).

Asimismo, España con 2.5 millones de hectáreas de olivar es líder mundial en producción de aceite de oliva (Ministerio de Agricultura, Alimentación y Medio Ambiente [Magrama], 20I4). En la campaña 2013-2014 la producción llegó a I.7 millones de toneladas, esto es, cerca de 50\% de la producción mundial (Consejo Oleícola Internacional, 20I4). En este país, el aceite de oliva es mucho más que un mero producto, es toda una cultura.

Tanto la producción de vino como la producción de aceite de oliva son ejecutadas, en su mayoría, por pequeñas y medianas empresas, muchas de estas de tipo cooperativas, y han generado riqueza suficiente para frenar, en parte, los flujos migratorios de las zonas 
rurales hacia las ciudades (López-Guzmán, Millán y Caridad, 2008). Las empresas de ambos sectores han ido cambiando su forma organizativa, y evolucionado hacia vinos y aceites de oliva extra virgen de mayor calidad (Martínez-Carrasco, Brugarolas y Del Campo, 2005), una tendencia que se ha mantenido en los últimos is años.

En el caso de los vinos, la calidad se garantiza por medio de las siguientes figuras', de mayor a menor calidad: a) Vinos de Calidad Producidos en una Región Determinada (Vcprd): vinos de pago, vinos con denominación de origen calificada, vinos con denominación de origen y vinos de calidad con indicación geográfica; y b) vinos de mesa.

En el caso del aceite de oliva extra virgen, la calidad se garantiza mediante la DOP o la IGP. Para obtener el estatus de DOP, el producto debe tener una calidad o características atribuibles, en esencia, al área local, mientras que para conseguir el estatus de IGP, basta que el aceite de oliva tenga una calidad o reputación específica atribuible a esa área.

De la superficie total destinada a la vid, cerca de 60\% está inscrita en alguna DO, donde se producen Vcprd con una tendencia al crecimiento, en detrimento de las superficies destinadas a vinos de mesa (Magrama, 20I4). España cuenta con 90 zonas de producción de Vcprd (I4 vinos de pago, 5 DO calificada, 64 DO y 7 vinos de calidad), y 4I clases de vinos de mesa con derecho a la mención tradicional "Vinos de la Tierra en España”. Respecto al aceite, España cuenta con 30 DOP de aceite de oliva virgen extra (Magrama, 20I4).

Como opción estratégica de desarrollo, muchas de estas denominaciones de origen, tanto en el caso del vino como del aceite de oliva, han impulsado la creación de rutas alimentarias. En lo que concierne al turismo del vino, se ha desarrollado una importante alianza estratégica (López-Guzmán, Rodríguez, Sánchez y Luján, 20II). La Secretaría General de Turismo (SGT), junto con la Asociación Española de Ciudades del Vino (Acevin), apostó por la creación de un producto turístico de calidad denominado Rutas del Vino, a raíz del cual se han certificado distintas iniciativas conforme a la marca Rutas del Vino de España.

En el año 200I, en una primera fase de implantación de este producto turístico de calidad, se proyectaron seis Rutas del Vino, entre las que se incluía MontillaMoriles. En fases posteriores se han incorporado nuevos destinos, de tal forma que, en este momento, se dispone de un Club de Producto turístico integrado por 20 rutas certificadas conforme a la marca Rutas del Vino de España. Del mismo modo,

I Ley 24/2003, de Io de julio, de la Viña y del Vino.

GENOVEVA MILLÁN VÁZQUEZ DE LA TORRE Y LEONOR M. PÉREZ * COMPARACIÓN DEL PERFIL DE ENOTURISTAS Y OLEOTURISTAS... 


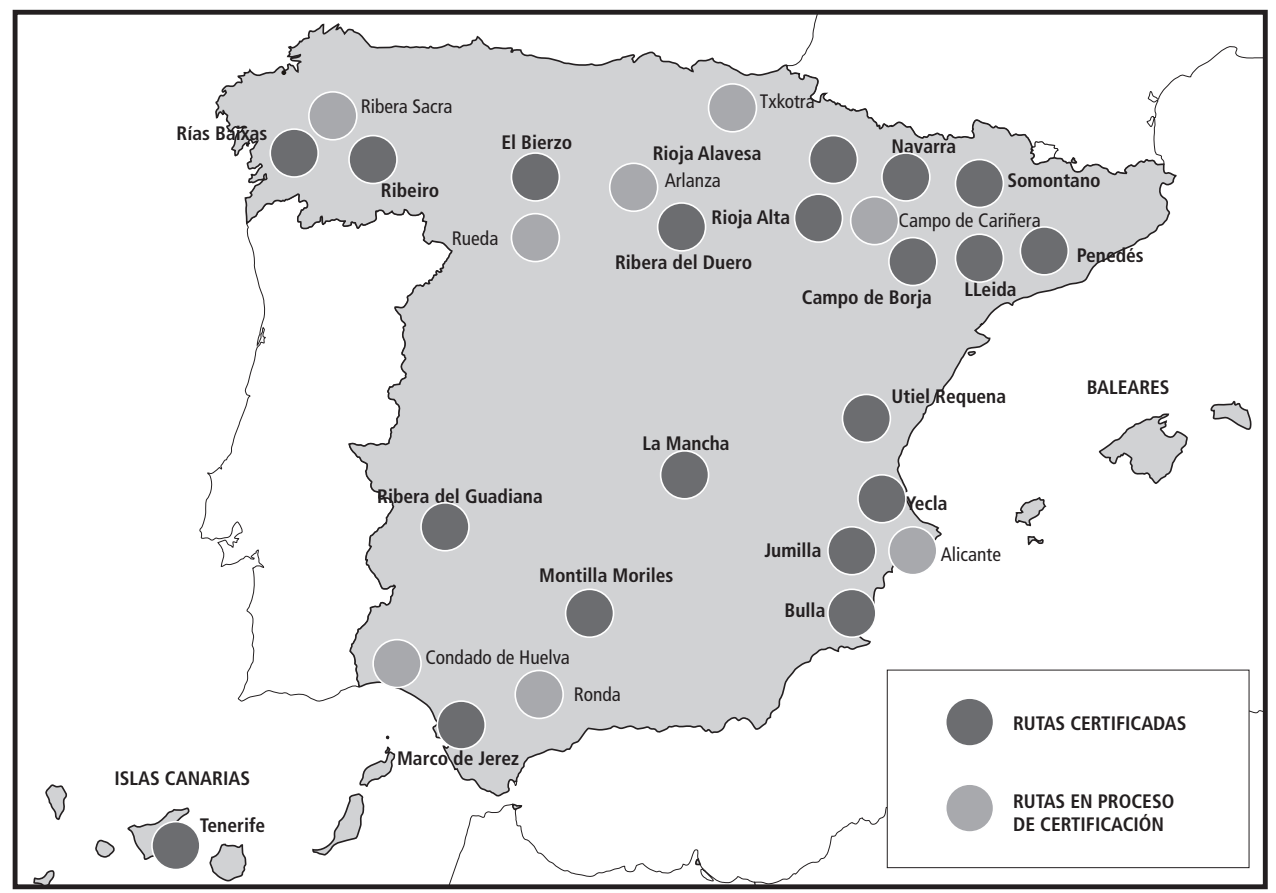

Figura i. Localización de las Rutas del Vino de España

Fuente: Acevin, 20I4

dentro del marco Acevin hay ocho rutas que están trabajando para certificarse y, por consiguiente, pasar a formar parte del Club de Producto (Acevin, 20I4) (ver Figura I).

En general, el turismo del vino en España se encuentra en una fase de desarrollo (Marzo y Pedraja, 20r2), aunque ya algunos destinos han alcanzado la fase de madurez. El número de enoturistas se ha incrementado $40 \%$ en los últimos cinco años: las bodegas han registrado un total de I 689209 visitantes durante el año 2013 (ver Figura 2). Las denominaciones de origen Penedés y Marco de Jerez son las que han atraído mayor número de viajeros, y las temporadas de mayor afluencia son otoño y primavera, lo que favorece la desestacionalización y el alargamiento de la temporada turística (Acevin, 20I4).

La práctica del oleoturismo ha emergido como un área similar a la práctica enoturística, y ha adquirido dentro del turismo industrial rural una alta potencialidad Molina, Quesada y Ruiz, 20II). Aunque el oleoturismo no ha recibido tanto apoyo como el enoturismo (Millán, Agudo y Morales, 20II), instituciones como la Asociación Española de Municipios del Olivo (AEMO) han impulsado su desarrollo, por tanto, hoy se destacan trece rutas a lo largo de la península (ver Figura 3). 


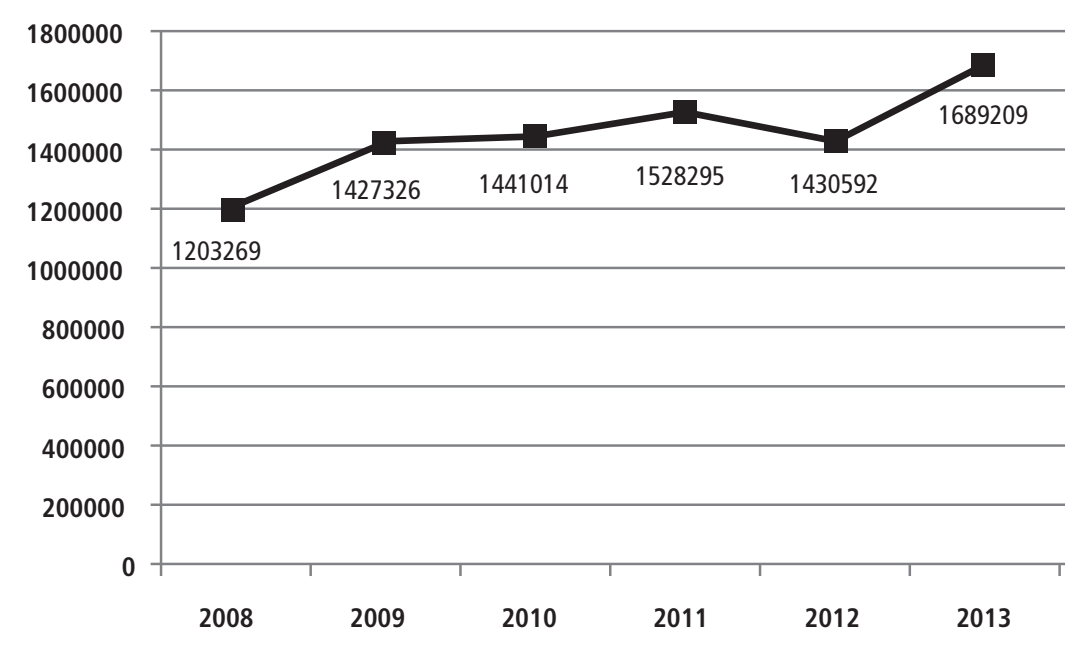

Figura 2. Evolución del número de visitantes en bodegas asociadas con las Rutas del Vino de España FuENTE: elaboración propia a partir de Acevin, 2014

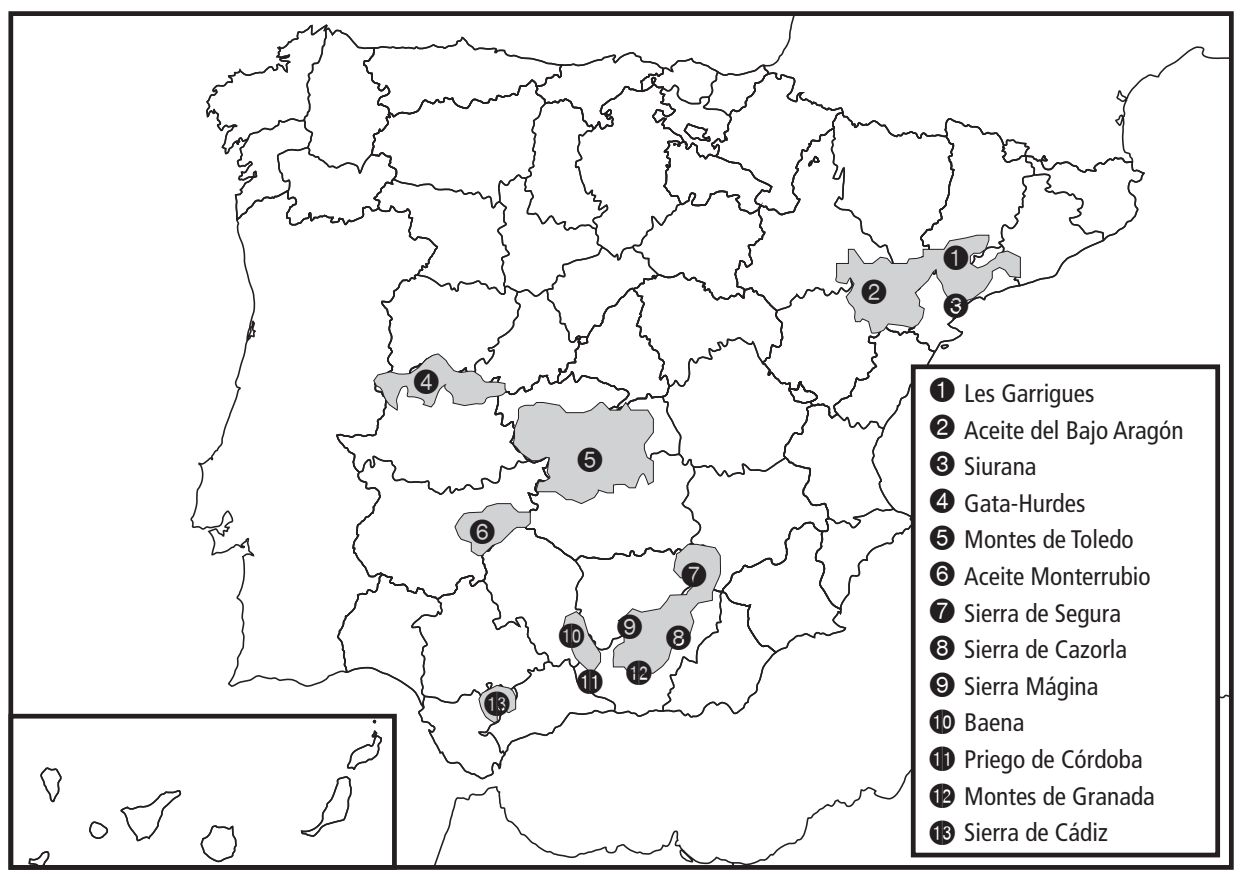

Figura 3. Localización de las principales rutas del aceite de oliva en España Fuente: Cerespain (2014) 
En general, el oleoturismo se encuentra en una fase de inicio. Los cálculos de demanda en regiones concretas (Millán, Arjona y Amador, 20I4) indican que el número de turistas no llega a superar los 150000 al año, aunque va en aumento. Así, dada la situación del oleoturismo en el último lustro, y de acuerdo con Molina, Quesada y Ruiz (20II), su desarrollo potencial podría vincularse de manera provechosa con otros itinerarios y rutas turísticas, cuya cooperación complemente la oferta turística. Como se observa en las figuras i y 3 , en algunas regiones existen rutas del vino y del aceite de oliva ubicadas en zonas muy próximas. De esta manera, si los perfiles de ambos tipos de turistas fuesen similares, se podrían lograr algunas sinergias.

\section{Rutas gastronómicas en Córdoba}

\section{I. Ruta del Vino Montilla-Moriles}

La Ruta del Vino Montilla-Moriles discurre por el sur de la provincia de Córdoba, atraviesa nueve municipios de la DO Montilla-Moriles, e incluye la capital. En estos municipios la actividad principal es la agricultura, seguida del sector servicios excepto en la capital, donde predomina el sector terciario. El sector secundario es prácticamente inexistente, excepto en la producción de vino y de aceite de oliva.

Esta ruta nació a principios de 200I, dentro de los compromisos adquiridos por el Ayuntamiento de Montilla, como ciudad del vino, con Acevin. En ese mismo año se constituyó la Asociación para la Promoción del Turismo del Vino (Avintur), cuyo fin primordial es la promoción y dinamización de la cultura del vino en el territorio de la DO Montilla-Moriles, con incidencia en aspectos turísticos derivados de la planificación y gestión de la ruta. El resto de municipios que hoy forma parte de la ruta, así como el Patronato Provincial de Turismo de Córdoba, se integraron posteriormente.

Tras un gran esfuerzo público-privado, en julio de 2005 se logró la certificación de la Ruta del Vino Montilla-Moriles como Ruta del Vino de España, la primera certificada de Andalucía. Por otra parte, los ayuntamientos que componen la ruta, la Consejería de Turismo y Deporte de la Junta de Andalucía y la SGT apostaron por un plan de dinamización turística que comenzó su ejecución en 2007.

Hoy la Ruta del Vino Montilla-Moriles cuenta con más de 50 compañías asociadas, entre estas, bodegas, lagares, alojamientos, restaurantes, empresas de ocio o agencias receptivas. El número de empresas vinculadas ha crecido desde su inicio, no obstante, la cifra es aún pequeña. Por ejemplo, solo 28.6\% de los operadores inscritos en el Consejo Regulador de la DOP forman parte de la ruta (Ruta del Vino Montilla-Moriles, 2014). 
La cantidad de enoturistas que visitan la ruta tuvo una tendencia creciente hasta el año 20II, y luego registró un descenso en los años siguientes (ver Figura 4). Se calcula que en 2013 hubo I2 353 visitantes en las bodegas, lo que supone un descenso de $23 \%$ respecto al año anterior, lo que significa volver a los niveles de demanda de 2008 (Acevin, 20I4). Estas cifras contrastan con los incrementos de demanda a escala nacional.

\subsection{Rutas del aceite de oliva en Córdoba}

El oleoturismo, aunque no ha recibido tanto apoyo como el enoturismo, también se ha ido desarrollando en la provincia de Córdoba desde principios del siglo XXI. Los entes públicos han elaborado programas para fomentar esta actividad, mediante proyectos comunitarios como Oleoturismo o Terra Olea. En 2005 el Patronato Provincial de Córdoba, en una apuesta para fomentar la cultura por el aceite de oliva virgen extra, creó ocho rutas gastronómicas vinculadas a tres DOP: Baena, MontoroAdamuz y Priego de Córdoba.

La DOP Baena abarca siete municipios, es la más antigua (fue la primera designación de origen que se creó en España en el sector agroalimentario, en 198I) y la más importante en el ámbito internacional, gracias a los premios obtenidos por la calidad de sus aceites. La DOP Montoro-Adamuz, la más reciente y menos conocida, incluye ocho municipios y aloja además varias reservas naturales como el Parque Natural Sierra de Cardeña Montoro. La DOP Priego de Córdoba, situada en el corazón del Parque Natural de las Sierras Subbéticas, cubre cuatro municipios, es la que recibe más visitas turísticas y cuenta con mayor infraestructura hotelera por su mayor desarrollo en turismo rural.

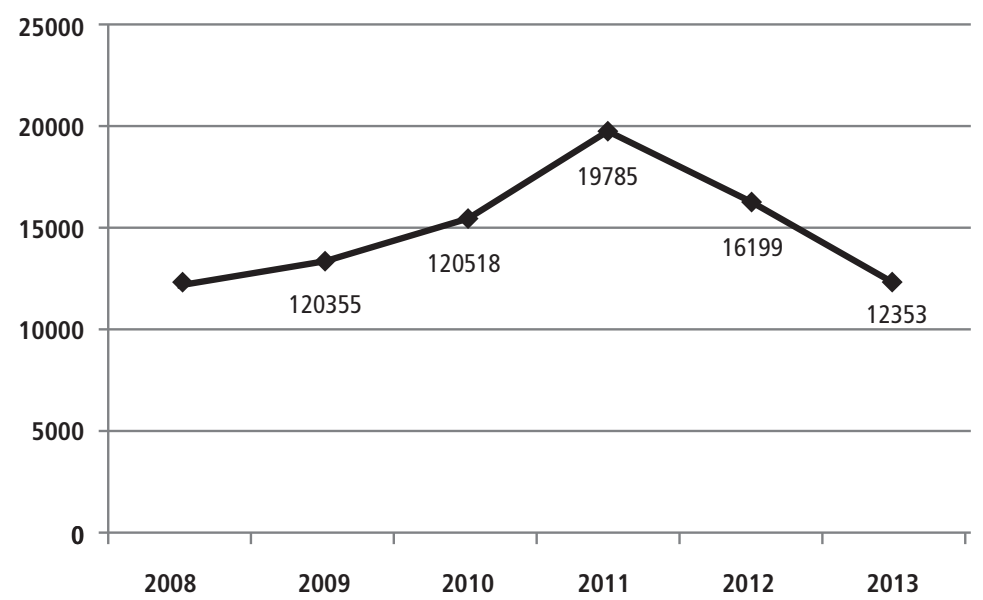

Figura 4. Evolución del número de visitantes en bodegas asociadas con la ruta Montilla-Moriles Fuente: elaboración propia a partir de Acevin, 2014 
En la actualidad, de las 50 almazaras que están inscritas en estas tres DOP, $40 \%$ no abre sus puertas para la visita de oleoturistas. Se trata de almazaras o molinos de aceite dedicados principalmente a la exportación, que no cuentan con el oleoturismo como una fuente adicional de ingresos. El resto de las almazaras (60\%), abren para recibir visitas pero con un horario bastante reducido: $33 \%$ solo abre por las mañanas, y solo $12 \%$ recibe turistas los fines de semana. El número de oleoturistas que han visitado estas rutas ha ido en alza en la última década. No obstante, no hay registros precisos de su cuantía.

En la Tabla i se resumen algunas características sobre el grado de desarrollo de las rutas analizadas, según las cuales, podemos considerar que las distintas rutas del aceite de oliva se encuentran en fase de desarrollo, dentro de su ciclo de vida como destinos de turismo gastronómico, aunque en estado incipiente si se compara con la ruta del vino Montilla-Moriles, mucho más consolidada. En concreto, al analizar las tres rutas del aceite, la de Baena es la que tiene un mayor nivel de desarrollo, seguida de Priego de Córdoba y, por último, Montoro-Adamuz.

TABLA 1. Resumen de las características de las rutas analizadas

\begin{tabular}{|c|c|c|}
\hline Características & Montilla-Moriles & Baena, Montoro-Adamuz y Priego de Córdoba \\
\hline $\begin{array}{l}\text { Escala del turismo } \\
\text { gastronómico }\end{array}$ & $\begin{array}{l}\text { Cierto número de } \\
\text { enoturistas; nuevas bodegas } \\
\text { adaptadas al turismo; } \\
\text { algunas han adquirido el } \\
\text { rango de monumento. }\end{array}$ & $\begin{array}{l}\text { Bajo número de oleoturistas; nuevas almazaras adaptadas } \\
\text { al turismo; algunas almazaras y olivos milenarios han } \\
\text { adquirido el rango de monumento en Baena y Priego de } \\
\text { Córdoba. En Baena existen museos dedicados al aceite. }\end{array}$ \\
\hline Tipos de turismo & $\begin{array}{l}\text { Crecimiento de la cantidad } \\
\text { de turistas atraídos por el } \\
\text { vino; algo de turismo más } \\
\text { convencional en bodegas } \\
\text { con rango de monumento. }\end{array}$ & $\begin{array}{l}\text { Crecimiento de la cantidad de turistas atraídos por } \\
\text { el aceite de oliva; algo de turismo más convencional } \\
\text { en las almazaras con rango de monumento. En } \\
\text { Montoro-Adamuz, el crecimiento de oleoturistas } \\
\text { es menor que en las otras dos rutas. }\end{array}$ \\
\hline $\begin{array}{l}\text { Importancia del } \\
\text { turismo para } \\
\text { bodegas/almazaras }\end{array}$ & $\begin{array}{l}\text { Mezcla de bodegas } \\
\text { orientadas al turismo } \\
(\mathrm{I} 8 \%) \text { y a la exportación. }\end{array}$ & $\begin{array}{l}\text { La orientación al turismo de las almazaras es } \\
\text { de } 68 \%, 57 \% \text { y } 53 \% \text { respectivamente en Baena, } \\
\text { Montoro-Adamuz y Priego de Córdoba. }\end{array}$ \\
\hline $\begin{array}{l}\text { Importancia } \\
\text { del turismo } \\
\text { gastronómico } \\
\text { para la región }\end{array}$ & $\begin{array}{l}\text { Se reconoce el potencial } \\
\text { del enoturismo, basado en } \\
\text { tendencias visibles; deseo } \\
\text { general para incrementarlo. }\end{array}$ & $\begin{array}{l}\text { Se reconoce el potencial del turismo del aceite, } \\
\text { aunque en menor grado que en el caso del vino; deseo } \\
\text { moderado pero creciente para incrementarlo. }\end{array}$ \\
\hline $\begin{array}{l}\text { Sinergias con otro } \\
\text { segmento turístico }\end{array}$ & $\begin{array}{l}\text { Se requieren medidas } \\
\text { políticas; el desafío } \\
\text { está en la resolución de } \\
\text { conflictos de intereses. }\end{array}$ & $\begin{array}{l}\text { Las tres rutas están próximas a la ruta Montilla-Moriles. } \\
\text { La de Baena es complementaria al enoturismo, } \\
\text { pues cuenta con algunas bodegas. La de Montoro- } \\
\text { Adamuz es complementaria al turismo rural, } \\
\text { con el Parque Natural Cardeña Montoro. } \\
\text { La de Priego de Córdoba cuenta con un gran } \\
\text { desarrollo del turismo rural (infraestructuras, } \\
\text { casas y hoteles rurales), y del turismo arqueológico } \\
\text { (cercana a zona arqueológica de Almedinilla). }\end{array}$ \\
\hline
\end{tabular}

Fuente: elaboración propia 


\section{Metodología}

Para analizar el perfil de los visitantes se desarrolló un trabajo de campo en que, mediante muestreo aleatorio simple, se encuestó tanto a enoturistas como a oleoturistas que permanecieron al menos seis horas en las rutas analizadas (ver Tabla 2). En concreto, se encuestó a 500 enoturistas que visitaron la DO Montilla-Moriles en los meses de marzo a mayo de 2006, y 2324 oleoturistas que visitaron las DOP Baena, Montoro-Adamuz o Priego de Córdoba entre septiembre de 2007 y octubre de $2008^{2}$.

TaBla 2. Ficha Técnica de las encuestas

\begin{tabular}{lll}
\hline & Enoturismo & Oleoturismo \\
\hline Población & $\begin{array}{l}\text { Turistas que visitaron la ruta } \\
\text { Montilla-Moriles (hombres y } \\
\text { mujeres, mayores de I8 años) }\end{array}$ & $\begin{array}{l}\text { Turistas que visitaron las rutas } \\
\text { de Baena, Montoro-Adamuz y } \\
\text { Priego de Córdoba (hombres y } \\
\text { mujeres, mayores de I8 años) }\end{array}$ \\
\hline Tamaño muestral & 500 encuestas válidas & 2324 encuestas válidas \\
\hline Error muestral & $\pm 4, \mathrm{I} \%$ & $\pm 3,7 \%$ \\
\hline Nivel de confianza & $95 \% ; \mathrm{p}=\mathrm{q}=0,5$ & $95 \% ; \mathrm{p}=\mathrm{q}=0,5$ \\
\hline Sistema de muestreo & Aleatorio & Aleatorio \\
\hline Fecha trabajo de campo & marzo - mayo 2006 & septiembre 2007 a octubre 2008 \\
\hline
\end{tabular}

Fuente: elaboración propia

Para diseñar el cuestionario se realizaron diversas entrevistas a los presidentes de los Consejos Reguladores de las denominaciones de origen, así como a empresarios vinculados con las rutas. Luego se realizó un pretest con 20 enoturistas y oleoturistas para su validación. El cuestionario final incorporó 35 preguntas estructuradas en seis bloques: perfil sociodemográfico, perfil económico, perfil del turista potencial, motivo del viaje, características del viaje y opiniones sobre la ruta.

Respecto al tratamiento de los datos, se realizó en primer lugar un análisis de frecuencias para las muestras de enoturistas y oleoturistas. Posteriormente, para contrastar si existían diferencias significativas entre los dos perfiles o si, por el contrario, ambas muestras independientes procedían de la misma población, se utilizó la prueba de Kruskal-Wallis. Se trata de un test no paramétrico alternativo al método Anova, que sirve para contrastar la hipótesis de que $k$ muestras provienen de la misma población, y no asume normalidad en los datos.

2 Los estudios corresponden a dos proyectos subvencionados por la Consejería de Turismo Comercio y Deporte de la Junta de Andalucía (2006/282754 y CO-007/08-FFI). 


\section{Perfil de enoturistas y oleoturistas}

La Tabla 3 resume algunas características de los turistas. Tanto en el caso del enoturismo como en el caso del oleoturismo predominan los visitantes del sexo masculino ( $53 \%$ y $55 \%$, respectivamente). Respecto a la edad, más de un tercio de enoturistas y oleoturistas (algo más de $38 \%$ ) se encuentra en el intervalo de 50 a 59 años. En cuanto al enoturismo, hay un mayor porcentaje de personas que superan los 60 años ( $7.7 \%)$ y menor entre 40 y 49 años (15.4\%), en comparación con la cantidad de oleoturistas ( $2.7 \%$ y $24.1 \%$, respectivamente). Sin embargo, no podemos afirmar que existan diferencias significativas entre la edad de ambos tipos de visitantes. Según la prueba de Kruskal-Wallis, ambas muestras son homogéneas, es decir, pertenecen a la misma población (sig. asintót. = 0,429).

Tampoco existen diferencias significativas entre el nivel de estudios de enoturistas y oleoturistas (sig. asintót. $=0,849$ ). La mayoría tiene estudios medios ( $93 \%$ de enoturistas y $89 \%$ de oleoturistas). Ahora bien, el poder adquisitivo de estos grupos es significativamente diferente (sig. asintót. $=0,008)$ : el enoturista suele tener mayor poder adquisitivo, $32 \%$ tiene una renta mensual superior a $2500 €$, mientras que tan solo $4 \%$ de los oleoturistas alcanza ese nivel de renta.

Por el contrario, la dedicación profesional es muy parecida; destacan las personas con empleo ( $55 \%$ y $53 \%$ de enoturistas y oleoturistas, respectivamente) y un pequeño porcentaje de estudiantes ( $7 \%$ y $5 \%$, respectivamente). En cuanto al estado civil, la mayoría están casados (55\% de enoturistas y 50\% de oleoturistas), mientras que los visitantes solteros representan $25 \%$ y $35 \%$, de forma respectiva.

Tan solo 3\% de enoturistas y I\% de oleoturistas proceden de otros países. Así, el turismo gastronómico de la provincia es una actividad desarrollada sobre todo por el turismo doméstico, en especial, de otras zonas de Andalucía. Por otra parte, la mayoría de los turistas del vino y del aceite de oliva realizan el viaje en familia (algo más de 40\%) o con su pareja (aproximadamente, $35 \%$ ); es menor el número de personas que realiza el viaje con amigos ( $15 \%$ de enoturistas y $12 \%$ de oleoturistas), y más bajo aún el número de turistas que viajan solos ( $8 \%$ de enoturistas y $5 \%$ de oleoturistas).

Sobre la duración en el destino no existen diferencias significativas entre ambos grupos (sig. asintót. = 0,257). La mayoría (50\% en el caso del enoturismo y $63 \%$ en el caso del oleoturismo) son excursionistas que están menos de 24 horas en la zona de la ruta y no consumen alojamiento, aunque sí el resto de servicios. Tan solo 10\% de enoturistas y $9 \%$ de oleoturistas permanecen más de tres días en el destino. 
TABla 3. Perfil de enoturistas y oleoturistas

\begin{tabular}{|c|c|c|c|}
\hline & & ENOTURISTAS (\%) & Oleoturistas (\%) \\
\hline \multirow{2}{*}{ Sexo } & Mujeres & 46.8 & $45 . \mathrm{I}$ \\
\hline & Hombres & 53.2 & 54.9 \\
\hline \multirow{5}{*}{ Edad } & I8-29 & 19.2 & 15.6 \\
\hline & $30-39$ & 19.2 & 18.2 \\
\hline & $40-49$ & 15.4 & 24.I \\
\hline & $50-59$ & 38.5 & 39.4 \\
\hline & 60 o más & 7.7 & 2.7 \\
\hline \multirow{4}{*}{ Estudios } & Sin estudios & 0.3 & 2.8 \\
\hline & Estudios primarios & 16.0 & 24.3 \\
\hline & Estudios secundarios & 77.0 & 64.8 \\
\hline & Estudios superiores & 6.7 & 8.1 \\
\hline \multirow{3}{*}{ Renta familiar } & Menos de 1500 & 40.3 & $45 \cdot 4$ \\
\hline & $1500-2500$ & 28.I & 50.3 \\
\hline & Más de 2500 & 31.6 & 4.3 \\
\hline \multirow{8}{*}{ Dedicación } & Profesional & 10.2 & 6.6 \\
\hline & Empresario & 3.1 & 2.9 \\
\hline & Directivo & 2.4 & 4.8 \\
\hline & Funcionario & I8.6 & 20.2 \\
\hline & Trabajador cualificado & 21.4 & 19.3 \\
\hline & Estudiante & 7.3 & 5.4 \\
\hline & Jubilado & 24.5 & 23.1 \\
\hline & Desempleado & I2.5 & 17.7 \\
\hline \multirow{3}{*}{ Estado civil } & Soltero & 24.9 & 35.3 \\
\hline & Casado & 55.1 & 50.4 \\
\hline & Otros & 20.0 & 14.3 \\
\hline
\end{tabular}




\begin{tabular}{|c|c|c|c|}
\hline \multirow{3}{*}{ Origen } & Andalucía & 71.2 & $8 \mathrm{I} .2$ \\
\hline & Resto de España & 25.2 & 17.6 \\
\hline & Otros países & 3.6 & I. 2 \\
\hline \multirow{4}{*}{ Con quién viaja } & Solo & 8 & 5.4 \\
\hline & En pareja & 35 & 34.7 \\
\hline & Con la familia & 42 & 47.6 \\
\hline & Con amigos & 15 & I2.3 \\
\hline \multirow{3}{*}{ Duración del viaje } & Menos de 24 horas & 50.4 & 62.8 \\
\hline & 2-3 días & 39.9 & 28.I \\
\hline & Más de 3 días & $9 \cdot 7$ & 9.I \\
\hline \multirow{3}{*}{ Gasto diario } & Menos de $35 €$ & I2.I & 27.3 \\
\hline & Entre $36-5 \circ €$ & 27.7 & 56.4 \\
\hline & Más de $5 \circ €$ & 60.2 & 16.3 \\
\hline \multirow{4}{*}{$\begin{array}{l}\text { Bodegas/almazaras } \\
\text { visitadas }\end{array}$} & Una & 61.5 & 60.4 \\
\hline & Dos & 27 & 27.2 \\
\hline & Tres & 7.7 & 8.4 \\
\hline & Más de tres & 3.8 & 4.0 \\
\hline \multirow{3}{*}{ Motivación } & Visitar la bodega/almazara & 59.8 & 51.3 \\
\hline & Conocer la comarca & 35.1 & 42.2 \\
\hline & Otros & 5.1 & 6.5 \\
\hline \multirow{4}{*}{ Elección de la Ruta } & Por recomendación de amigos & 58.6 & 74.3 \\
\hline & Por internet & 23.1 & 15.6 \\
\hline & Agencia de viajes & 5.2 & 2.1 \\
\hline & $\begin{array}{l}\text { Anuncios en medios } \\
\text { de comunicación }\end{array}$ & 13.1 & 8.0 \\
\hline \multirow{2}{*}{ Repetiría la visita } & Sí & 79.6 & 48.4 \\
\hline & No & 20.4 & 51.6 \\
\hline \multirow{3}{*}{ Satisfacción } & Satisfecho & 75.6 & 69.9 \\
\hline & Indiferente & I8.9 & 23.1 \\
\hline & Insatisfecho & 5.5 & 7.0 \\
\hline \multirow{2}{*}{ Recomendaría la ruta } & $\mathrm{Si}$ & 84 & 52 \\
\hline & No & I6 & 48 \\
\hline
\end{tabular}

Fuente: elaboración propia 
El impacto económico que genera el enoturista en la zona de la ruta es significativamente diferente del impacto que genera el oleoturista (sig. asintót. = 0 ). La mayoría de enoturistas (60\%) gasta más de 50 euros diarios en la ruta, mientras que la mayoría de oleoturistas ( $56 \%$ ) suelen gastar entre 36 y 50 euros cada día.

En lo que corresponde al número de bodegas y almazaras visitadas, no existen diferencias significativas entre los dos perfiles (sig. asintót. = 0,863). La mayoría en ambos tipos de visitantes (algo más de 60\%) tan solo conocen una bodega o una almazara. En los dos casos, esas visitas suelen hacerse mediante viajes organizados. El número de turistas que visita dos bodegas o almazaras está alrededor de $27 \%$, y son muy pocos los que visitan tres ( $7.7 \%$ de enoturistas y $8.4 \%$ de oleoturistas) o más de tres (no más de $4 \%$ en ambos casos). En concreto, son aquellos que viajan en coche privado los que suelen visitar más bodegas y almazaras. Sin embargo, cabe destacar que en cuanto a la motivación principal, en ambos tipos de visitantes destaca el visitar las bodegas $(60 \%)$ o almazaras ( $51 \%)$ frente a conocer la comarca ( $35 \%$ y $42 \%$, respectivamente).

Por otro lado, la mayoría de los enoturistas y oleoturistas que han realizado alguna de las rutas ha mostrado su interés tanto en repetir la visita como su grado de satisfacción, aunque han identificado algunas deficiencias como la falta de actividades complementarias, el alto precio del viaje o la falta de profesionalización del sector en cuanto a la atención al visitante en bodegas y almazaras.

Se evidencia que la DO del vino Montilla-Moriles es más conocida y visitada por el turista gastronómico que las DOP del aceite de oliva. Un 75\% de los oleoturistas conocía (por referencia o por haberla visitado) la DO del vino Montilla-Moriles, mientras que solo I2 \% conocía alguna de las tres DOP de aceite de oliva.

Por último, se encontró que tanto la demanda de enoturismo como la de oleoturismo se caracterizan por una estacionalidad que varía de forma cíclica cada doce meses; sus puntos máximos ocurren en Navidad, en las vacaciones de verano, Semana Santa y puentes. En esas fechas la ocupación hotelera de las distintas zonas analizadas suele rondar $80 \%$. En las fechas de Navidad (sobre todo en Nochevieja) esta ocupación alcanza 100\%, y deja buena parte de la demanda sin atender por falta de oferta de alojamiento.

\section{Conclusiones}

La revisión de la literatura pone de manifiesto cómo las rutas gastronómicas basadas en productos agrarios de alta calidad podrían favorecer el desarrollo de zonas 
rurales. En estas iniciativas, el establecimiento de alianzas entre diferentes actores desempeña un papel estratégico, según Telfer (200I). Sin embargo, si en una región hay rutas basadas en productos agrarios diferentes, ¿se podrían establecer alianzas entre estas? Si hubiera semejanzas entre los perfiles de los turistas en ambas rutas, se podrían desarrollar de forma activa vínculos centrados en las necesidades del consumidor turístico y realizar acciones de promoción conjunta.

En España hay muchas regiones donde se han creado rutas turísticas enfocadas en el vino y en el aceite de oliva. Así, este artículo realiza una comparación entre el perfil de enoturistas y oleoturistas en varias rutas de la provincia de Córdoba ligadas a la DOP para identificar semejanzas. La originalidad de este estudio se centra en la necesidad de profundizar en la demanda de enoturismo y oleoturismo en España, así como en la falta de trabajos de investigación que cotejen ambos perfiles.

Los resultados muestran que existen diferencias y semejanzas entre el perfil de enoturistas y oleoturistas. Sus perfiles demográficos, su forma de organizar el viaje, su comportamiento en el destino y los determinantes de su satisfacción son parecidos, pero no sus perfiles económicos y el gasto que realizan en el destino. El enoturista suele tener mayor poder adquisitivo y realiza un mayor gasto a lo largo de la ruta.

Las semejanzas entre enoturismo y oleoturismo ponen de manifiesto que se podrían desarrollar de forma activa vínculos centrados en las necesidades del consumidor, y realizar acciones de promoción conjunta. Es decir, las distintas industrias (vino, aceite y turismo) podrían trabajar de forma colectiva y compartir inversiones, recursos y conocimiento para favorecer el desarrollo rural. Esto es importante, en particular para el oleoturismo, puesto que no recibe tanto apoyo para su desarrollo.

Por ejemplo, se podría compartir inversión para profesionalizar el sector, en cuanto a mejorar la atención de los turistas en bodegas y almazaras. Un trabajador que moltura el aceite sin un curso específico de atención al cliente, quizás no sea la persona más adecuada para explicar el proceso productivo. Ese trabajador debe ser capaz de transmitir el valor de los productos, la tierra, el entorno histórico y sociocultural que les rodea, así como generar la fidelización del turista.

Es interesante recordar que la demanda de turismo del vino y del aceite de oliva crece a escala nacional; además, hay una alta probabilidad de que el turista repita la experiencia, lo cual implica que las empresas podrían tener un futuro prometedor si realizan más inversiones en esta área con la finalidad de satisfacer este segmento turístico.

Puesto que las bodegas y almazaras son el principal atractivo de estas rutas, es importante que la zona disponga de un número suficiente de establecimientos abiertos de este tipo, sobre todo en fines de semana y periodos de vacaciones. 
La estacionalidad de la demanda se podría amortiguar al exportar las rutas a otras comunidades autónomas u otros países, y aprovechar así las oportunidades de viaje en distintas fechas, dada la diversidad de fiestas locales. Las zonas analizadas se encuentran relativamente bien comunicadas, por carretera y ferrocarril, y están muy cerca de dos aeropuertos internacionales, lo cual es un elemento decisivo para que ciudadanos de otros países consuman estos productos turísticos.

Asimismo, la mayoría de enoturistas y oleoturistas lo hace por medio de viajes organizados y tan solo visitan una bodega o una almazara, es decir, no necesitan pernoctar, por tanto, podría ser interesante que los viajes organizados establecieran rutas con visitas a un mayor número de bodegas o almazaras, para propiciar que los visitantes decidieran pasar la noche en estas zonas. Esta medida debería ir acompañada, por supuesto, de una mayor oferta de plazas hoteleras y casas rurales.

Otra medida que podría incrementar la demanda de alojamiento sería la cooperación para crear alguna actividad nocturna, desde exquisitas cenas hasta festivales gastronómicos. Además, tanto en enoturismo como en oleoturismo los turistas se quejan de la falta de actividades complementarias.

Los resultados de esta investigación pueden contribuir a que los destinos que deseen fomentar simultáneamente las actividades turísticas de enoturismo y oleoturismo puedan diseñar planes estratégicos eficaces. El futuro de las rutas gastronómicas en España es prometedor, con oportunidades para diversificar la oferta, romper con la estacionalidad y desarrollar nuevos destinos en un país que se caracteriza por la importancia de su sector turístico. Sin duda, acciones cooperativas entre la industria del vino, del aceite y del turismo beneficiaría el desarrollo sostenible de estas zonas rurales.

\section{Referencias}

Armesto, X. y Gómez, B. (2004). Productos agroalimentarios de calidad, turismo y desarrollo local: el caso del Priorat. Cuadernos Geográficos, 34(I), 83-94.

Asociación Española de Ciudades del Vino [Acevin] (20I4). Recuperado el I4 de junio de 20I4, de http://www.acevin.es

Briedenhann, J. y Wickens, E. (2004). Tourism Routes as a Tool for the Economic Development of Rural Areas. Vibrant Hope or Impossible Dream? Tourism Management, 25(I), 7I-79.

Bruwer, J. (2003). South African Wine Routes: Some Perspectives on the Wine Tourism Industry's Structural Dimensions and Wine Tourism Product. Tourism Management, 24(4), 423-435.

genoveva millán vázquez de la torre y leonor m. pérez * comparación del perfil de enoturistas y oleoturistas... 
Bruwer, J. y Alant, K. (2009). The Hedonic Nature of Wine Tourism Consumption: an Experiential View. International Journal of Wine Business Research, $2 I(3), 235-257$.

Cerespain (2014). Rutas turísticas por las zonas de producción del aceite de oliva virgen extra. Recuperado el I4 de junio de 20I4, de http://www.cerespain.com/rutas_ del_aceite.html

Charters, S. y Ali-Knight, J. (2002). Who is the Wine Tourist? Tourism Management, 23(3), 3II-319.

Charters, S. y Spielmann, N. (2014). Characteristics of Strong Territorial Brands: the Case of Champagne. Journal of Business Research, 67(7), 1461-1467.

Clemente, J., Rodríguez, J. y Buitrago, J. (2010). Análisis del mercado potencial enoturístico en Valencia. Papers de Turisme, 47-48, 93-108.

Consejo Oleícola Internacional (2014). Recuperado el I4 de junio de 20I4, de http://www.internationaloliveoil.org/estaticos/view/I3I-world-olive-oil-figures

Díaz, R. (2008). Potencialidad e integración del turismo del vino en un destino de sol y playa: el caso de Tenerife. Pasos, Revista de Turismo y Patrimonio Cultural, 6(2), I99-2I2.

Dodd, T. (1999). Attracting Repeat Customers to Wineries. International Journal of Wine Marketing, II(2), I8-28.

Duarte, A. (2010). Olives, Hospitality and Tourism: a Western Australian Perspective. British Food Journal, II2(I), 55-68.

Fondo Español de Garantía Agraria (20I4). Recuperado el I4 de junio de 20I4, de http://www.fega.es/PwfGcp/buscar?pagina_resultado=/es/buscador/resultado_ agrupacion_documentos.jsp\&pd=S\&ptd=95\&publicacion=tcm:0-5-I\&S=58\&destin $\mathrm{o}=$ Extranet;\&maxCodigoSeguridad $=0$

Gilg, A. y Battershill, M. (1998). Quality Farm Food in Europe: a Possible Alternative to the Industrialised Food Market and to Current AgriEnvironmental Policies: Lessons from France. Food Policy, 23(I), 25-40.

Hall, C. (2005a). Rural Wine and Food Tourism Cluster and Network Development. En D. Hall, I. Kirkpatrick y M. Mitchell (Eds.), Rural and Sustainable Business (pp. I49-64). Clevendon: Channel View.

Hall, C. (2005b). Biosecurity and Wine Tourism. Tourism Management, 26(6), 931-938. Hojman, D. y Hunter-Jones, P. (2012). Wine Tourism: Chilean Wine Regions and Routes. Journal of Business Research, 65(I), 13-2I.

Ilbery, B. y Kneafsey, M. (2000). Producer Constructions of Quality in Regional Speciality Food Production: a Case Study from South West England. Journal of Rural Studies, I6(2), 217-230. 
Kivela, J. y Crotts, J. (2006). Tourism and Gastronomy: Gastronomy's Influence on How Tourists Experience a Destination. Journal of Hospitality \&- Tourism Research, 30(3), 354-377.

López Guzmán, T., Millán, G. y Caridad, J. (2008). Análisis econométrico del enoturismo en España. Un estudio de caso. Estudios y Perspectivas en Turismo, I7 (2), 98-II

López-Guzmán, T., Rodríguez, J., Sánchez, S. y Luján, M. (20II). The Development of Wine Tourism in Spain. International Journal of Wine Business Research, 23(4), $374-386$

López Guzmán, T., Rodríguez, J. y Vieira, A. (2013). Revisión de la literatura científica sobre enoturismo en España. Cuadernos de Turismo, 32(I), I7I-188.

López-Guzmán, T., Vieira, A. y Rodríguez, J. (2014). Profile and Motivations of European Tourists on the Sherry Wine Route of Spain. Tourism Management Perspectives, ${ }_{I I}(\mathrm{I}), 63-68$

Macionis, N. y Cambourne, B. (1998). Wine and Food Tourism in the Australian Capital Territory: Exploring the Links. International Journal of Wine Marketing, Io(3), 5-22.

Martínez-Carrasco, L., Brugarolas, M. y Del Campo, F. (2005). Vinos de calidad poco conocidos: estimación de su potencial de mercado. Revista Europea de Dirección y Economía de la Empresa, I4(4), I39-156.

Marzo, M. y Pedraja, M. (2009). Wine Tourism Development from the Perspective of the Potential Tourist in Spain. International Journal of Contemporary Hospitality Management, $2 I(7), 816-835$.

Marzo, M. y Pedraja, M. (20I0). Are There Different Profiles of Wine Tourists? An Initial Approach. International Journal of Wine Business Research, 22(4), 349-36r.

Marzo, M. y Pedraja, M. (2012). Critical Factors of Wine Tourism: Incentives and Barriers from the Potential Tourist's Perspective. International Journal of Contemporary Hospitality Management, 24(2), 312-334.

Millán, G., Agudo, E. y Morales, E. (20II). Análisis de la oferta y la demanda de oleoturismo en el sur de España: un estudio caso. Cuadernos de Desarrollo Rural, $8(67), \mathrm{I} 8 \mathrm{I}-202$.

Millán, G., Arjona, J. y Amador, L. (20I4). A New Market Segment for Olive Oil: Olive Oil Tourism in the South of Spain. Agricultural Sciences, $5(3)$, 179-185.

Ministerio de Agricultura, Alimentación y Medio Ambiente [Magrama] (20I4). Recuperado el I4 de junio de 20I4, de http://www.magrama.gob.es/es/alimentacion/ temas/calidad-agroalimentaria/calidad-diferenciada/dop/default.aspx 
Miranda, B. y Fernández, B. (20II). Vino, turismo e innovación: las rutas del vino de España, una estrategia integrada de desarrollo rural. Estudios de Economía Aplicada, 29(I), I29-I64.

Mitchell, R. y Hall, C. (2004). The Post-Visit Consumer Behavior of New Zealand Winery Visitors. Journal of Wine Research, $15(\mathrm{I}), 39-49$.

Mitchell, R. y Hall, C. (2006). Wine Tourism Research: the State of Play. Tourism Review International, 9(4), 307-332.

Molina, V., Quesada, J. y Ruiz, I. (20II) Potencial del oleoturismo como diversificación económica del sector cooperativo agrario: el caso español. Revista de Ciencias Sociales, $17(3)$, 533-54I.

Organización Internacional de la Viña y el Vino (2014). Recuperado el I4 de junio de 20I4, de http://www.oiv.int/oiv/info/espublicationsstatistiques

Okumus, B., Okumus, F. y McKercher, B. (2007). Incorporating Local and International Cuisines in the Marketing of Tourism Destinations: the Cases of Hong Kong and Turkey. Tourism Management, 28(I), 253-26I.

Ruta del Vino Montilla-Moriles (20I4). Recuperado el I4 de junio de 20I4, de http://www.rutadelvinomontillamoriles.com/descargas/dossier_prensa_ montillamoriles.pdf

Scherrer, P., Alonso, A. y Sheridan, L. (2009). Expanding the Destination Image: Wine Tourism in the Canary Islands. International Journal of Tourism Research, II(5), 45I-463. Telfer, D. (2000). Tastes of Niagara: Building Strategic Alliances between Tourism and Agriculture. International Journal of Hospitality and Tourism Administration, I(I), 7I-88. Telfer, D. (200I). Strategic Alliances along the Niagara Wine Route. Tourism Management, 22(I), 2I-30.

Tomljenovic, R. y Getz, D. (2009). Life-Cycle Stages in Wine Tourism Development: a Comparison of Wine Regions in Croatia. Tourism Review International, I3(I), 3I-49. Vargas, A., Porras, N., Plaza, M. y Riquel, F. (2008). Turismo enológico: comportamiento del turista y percepción de la población residente. Papers de Turisme, 43-44, 97-III. 